\title{
Números Tribonacci, S-unidades y triplas diofánticas
}

\author{
Carlos Alexis Gómez Ruiz* \\ Universidad del Valle, Departamento de Matemáticas, Cali, Colombia.
}

Resumen. La sucesión Tribonacci $T:=\left\{T_{n}\right\}_{n>0}$ tiene valores iniciales $T_{0}=T_{1}=0, \quad T_{2}=1$ y cada término posterior es la suma de los tres términos precedentes. En este artículo, estudiamos la ecuación $T_{n}=k T_{m}$, donde $k$ es una $S$-unidad, para un conjunto finito $S$ de primos. Particularmente, mostramos que cualquier par de miembros de la tripla diofántica $\{9,56,103\}$ asociada a $T+1$, no se puede extender a otra tripla diofántica asociada a $T+1$.

Palabras clave: Números Tribonacci, triplas diofánticas, formas lineales en logaritmos de números algebraicos.

MSC2010: 11B37, 11B39, 11J86.

\section{Tribonacci numbers, S-units and diophantine triples}

\begin{abstract}
The Tribonacci sequence $T:=\left\{T_{n}\right\}_{n \geq 0}$ has initial values $T_{0}=$ $T_{1}=0, T_{2}=1$ and each term afterwards is the sum of the preceding three terms. In this paper, we study the equation $T_{n}=k T_{m}$, where $k$ is an $S$-unit, for a finite set $S$ of primes. In particular, we show that any two members of the diophantine triple $\{9,56,103\}$ associated to $T+1$, can not be extended to other diophantine triple associated to $T+1$.

Keywords: Tribonacci numbers, diophantine triples, linear forms in logarithms of algebraic numbers.
\end{abstract}

\section{Introducción}

La sucesión de Fibonacci $\left\{F_{n}\right\}_{n \geq 0}$, dada por

$$
F_{n+2}=F_{n+1}+F_{n} \quad \text { y } \quad F_{0}=0, F_{1}=1,
$$

*E-mail: carlos.a.gomez@correounivalle.edu.co

Recibido: 01 de julio de 2015, Aceptado: 04 de agosto de 2015.

Para citar este artículo: C.A. Gómez Ruiz, Números Tribonacci, S-unidades y triplas diofánticas, Rev.

Integr. Temas Mat. 33 (2015), No. 2, 121-133. 
está históricamente asociada con el crecimiento poblacional de conejos. Sólo hasta el siglo XIX la sucesión de Fibonacci atrajo el interés de la comunidad matemática. El francés Edouard Lucas [23] fue autor de un extenso análisis, mostrando que la sucesión es un mundo abierto de curiosidades. Hoy en día la sucesión de Fibonacci tiene su lugar propio en la teoría de números al punto de contar con una sociedad de matemáticos, Fibonacci Association, dedicada a su estudio.

La sucesión de Fibonacci cuenta con una gran cantidad de generalizaciones. En este artículo centramos nuestra atención en una generalización estudiada por Spikerman [25], la cual presentamos a continuación.

La sucesión Tribonacci $T:=\left\{T_{n}\right\}_{n \geq 0}$ se inicia con los valores $T_{0}=T_{1}=0, T_{2}=1, \mathrm{y}$ cada término a continuación es la suma de los anteriores tres términos:

$$
T_{n+3}=T_{n+2}+T_{n+1}+T_{n}, \quad \text { para todo } n \geq 0 .
$$

Llamaremos a $T_{n}$ el $n$-ésimo número Tribonacci. La sucesión Tribonacci tiene asociado el polinomio $\Psi(x)=x^{3}-x^{2}-x-1$, al cual llamamos su polinomio característico. Este tiene una raíz real,

$$
\alpha=\frac{1}{3}\left(1+(19-3 \sqrt{33})^{1 / 3}+(19+3 \sqrt{33})^{1 / 3}\right),
$$

y dos raíces complejas conjugadas:

$$
\beta=\alpha^{-1 / 2} e^{i \theta} \quad \text { y } \quad \gamma=\alpha^{-1 / 2} e^{-i \theta}, \quad \text { con } \quad \theta \in(\pi / 2, \pi) .
$$

Notemos que $\alpha$ es cercana a 2 , mientras que $|\beta|=|\gamma|=\alpha^{-1 / 2}<1$.

Spickerman mostró en [25], que las raíces del polinomio característico $\Psi(x)$ juegan un papel central en la representación de los números Tribonacci:

$$
T_{n}=\frac{\alpha}{(\alpha-\beta)(\alpha-\gamma)} \alpha^{n}+\frac{\beta}{(\beta-\alpha)(\beta-\gamma)} \beta^{n}+\frac{\gamma}{(\gamma-\alpha)(\gamma-\beta)} \gamma^{n},
$$

para todo $n \geq 1$. Recientemente, Drenden y Du [7] mostraron que la representación anterior es equivalente a

$$
T_{n}=d_{\alpha} \alpha^{n-1}+d_{\beta} \beta^{n-1}+d_{\gamma} \gamma^{n-1},
$$

donde $d_{z}:=(z-1) /(4 z-6)$. Más aún, Dresden y Du mostraron que la contribución de $\beta$ y $\gamma$, en la representación (1), es muy pequeña. En efecto

$$
T_{n}=d_{\alpha} \alpha^{n-1}+e_{n}, \quad \text { con }\left|e_{n}\right|<1 / 2 .
$$

Una propiedad fundamental en nuestro trabajo es la siguiente:

$$
\alpha^{n-2} \leq T_{n} \leq \alpha^{n-1}, \quad \text { para todo } n \geq 1 .
$$

Esta desigualdad se debe a Bravo y Luca [5]. En la sección 3 usaremos el grupo de Galois de $\mathbb{Q}(\alpha, \beta)$ sobre $\mathbb{Q}$, el cual denotamos por $G:=\operatorname{Gal}(\mathbb{Q}(\alpha, \beta) / \mathbb{Q})$. Notemos que $G \cong S_{3}$. 
En este artículo investigamos sobre el cociente de números Tribonacci que son $S$-unidades. Dado un conjunto de números primos $S=\left\{p_{1}, p_{2}, \ldots, p_{s}\right\}$, consideramos los números racionales de la forma

$$
p_{1}^{a_{1}} p_{2}^{a_{2}} \cdots p_{s}^{a_{s}}, \quad \text { con } \quad a_{1}, a_{2}, \ldots, a_{s} \in \mathbb{Z} .
$$

Llamaremos a estos números, $S$-unidades.

En otras palabras, estudiaremos la ecuación diofántica

$$
T_{n}=p_{1}^{a_{1}} p_{2}^{a_{2}} \cdots p_{s}^{a_{s}} T_{m}, \quad \text { con } \quad n>m \geq 2 \quad \text { y } \quad a_{1}, \ldots, a_{s} \in \mathbb{Z} .
$$

Nuestro trabajo reciente en conjunto con F. Luca (ver [14]), nos permite garantizar que la ecuación diofántica (4) tiene una cantidad finita de soluciones $\left(n, m, a_{1}, \ldots, a_{s}\right)$, donde

$$
\operatorname{máx}\left\{n, m,\left|a_{1}\right|, \ldots,\left|a_{s}\right|\right\} \leq \operatorname{máx}\left\{10^{10^{9}}, \exp \left(p_{s}^{5}\right)\right\} .
$$

Notemos que para cualquier conjunto $S$ la cota superior dada arriba es demasiado grande para determinar las soluciones de la ecuación diofántica (4), aún computacionalmente. Además, la evidencia computacional nos muestra que, aunque el método de reducción mediante el algoritmo LLL corre en tiempo polinomial (ver la Sección 2.3.4 en [6]), esto nos podría tomar demasiado tiempo para reducir una cota del orden de $10^{10^{9}}$ o superior.

De manera más precisa, mostramos el siguiente resultado.

Teorema 1.1. Sea $n>m \geq 2$ y $a_{1}, \ldots, a_{s} \in \mathbb{Z}$. Si $\left(n, m, a_{1}, \ldots, a_{s}\right)$ es una solución de (4), entonces existe una constante $C_{s}$ efectivamente calculable, tal que

$$
\operatorname{máx}\left\{n, m,\left|a_{1}\right|, \ldots,\left|a_{s}\right|\right\}<2,2 \times 10^{20} C_{s} .
$$

Resultado numérico. Si $S=\{2,3,7,103\}$, entonces la ecuación diofántica (4) tiene 55 soluciones, donde cada solución cumple que

$$
\operatorname{máx}\left\{n, m,\left|a_{1}\right|, \ldots,\left|a_{4}\right|\right\} \leq 18 .
$$

En la Sección 2 presentamos nuestra herramienta principal, un teorema de Matveev [24], sobre una cota inferior para formas lineales en logaritmos de números algebraicos. En la Sección 3 demostramos el Teorema 1.1. En la Sección 4 determinamos todos los cocientes de números Tribonacci que son $S$-unidades, con primos 2, 3, 7, 103. En la Sección 5 usamos los resultados de la Sección 4, para mostrar que ningún par de miembros de la tripla diofántica $\{9,56,103\}$, asociada a la sucesión $T+1$ (ver [16]), puede extenderse a una nueva tripla diofántica asociada a la sucesión $T+1$.

Finalizamos dando una breve descripción del método de resolución que usamos. Primero, usamos cotas inferiores para formas lineales en logaritmos de números algebraicos a fin de hallar una cota superior para el máximo de las variables enteras de nuestra ecuación diofántica. Para el resultado numérico usamos un procedimiento que involucra retículos, el algoritmo LLL y un resultado de geometría de números (ver [6]), con el fin de reducir las cotas superiores a casos que podamos verificar computacionalmente. 


\section{Formas lineales en logaritmos}

Sea $\eta$ un número algebraico de grado $d$ sobre $\mathbb{Q}$, con polinomio primitivo minimal sobre los enteros

$$
f(X):=a_{0} \prod_{i=1}^{d}\left(X-\eta^{(i)}\right) \in \mathbb{Z}[X],
$$

donde el coeficiente principal $a_{0}$ es positivo. Definimos la altura logarítmica de $\eta$ como

$$
h(\eta):=\frac{1}{d}\left(\log a_{0}+\sum_{i=1}^{d} \log \operatorname{máx}\left\{\left|\eta^{(i)}\right|, 1\right\}\right) .
$$

Nuestra herramienta principal es el siguiente teorema de Matveev [24]:

Teorema 2.1. Sean $\mathbb{K}$ un cuerpo de números algebraicos real de grado $D$ sobre $\mathbb{Q}$, $\eta_{1}, \ldots, \eta_{t}$ elementos de $\mathbb{K}$, y $b_{1}, \ldots, b_{t}$ enteros. Tomemos

$$
\Lambda:=\eta_{1}^{b_{1}} \cdots \eta_{t}^{b_{t}}-1 \quad \text { y } \quad B \geq \operatorname{máx}\left\{\left|b_{1}\right|, \ldots,\left|b_{t}\right|\right\} .
$$

Sean además $A_{i} \geq \operatorname{máx}\left\{D h\left(\eta_{i}\right),\left|\log \eta_{i}\right|, 0,16\right\}$ números reales, para $i=1, \ldots, t$. Entonces, asumiendo que $\Lambda \neq 0$, tenemos que

$$
|\Lambda|>\exp \left(-1,4 \times 30^{t+3} \times t^{4,5} \times D^{2}(1+\log D)(1+\log B) A_{1} \cdots A_{t}\right) .
$$

\section{Demostración del Teorema 1.1}

Primero que todo, notemos que:

- Para cada $1 \leq i \leq s$, tenemos que $p_{i}^{\left|a_{i}\right|}$ divide a $T_{n}$ ó $T_{m}$. Así, en virtud de la desigualdad (3) y del hecho que $p_{i} \geq 2>\alpha$, podemos concluir que

$$
\operatorname{máx}\left\{\left|a_{i}\right|: 1 \leq i \leq s\right\}<n .
$$

- Dado que $n>m$, entonces $p_{1}^{a_{1}} p_{2}^{a_{2}} \cdots p_{s}^{a_{s}}>1$.

Como una consecuencia de la primera observación, concluimos que una cota superior para $n$ será una cota superior para las demás variables enteras. Es decir,

$$
\operatorname{máx}\left\{n, m, k,\left|a_{1}\right|, \ldots,\left|a_{s}\right|\right\} \leq n .
$$

Nuestro primer paso consiste en mostrar que $m<C \log n$, para una constante $C>0$ efectivamente calculable. Usando el estimativo (2) de Dresden y Du en la igualdad (4), tenemos que

$$
\left|d_{\alpha} \alpha^{n-1}-p_{1}^{a_{1}} p_{2}^{a_{2}} \cdots p_{s}^{a_{s}} d_{\alpha} \alpha^{m-1}\right|<\frac{1}{2}\left(1+p_{1}^{a_{1}} p_{2}^{a_{2}} \cdots p_{s}^{a_{s}}\right) .
$$

Dividimos ambos lados de la desigualdad anterior por $p_{1}^{a_{1}} p_{2}^{a_{2}} \cdots p_{s}^{a_{s}} d_{\alpha} \alpha^{m-1}$ para obtener

$$
\left|p_{1}^{-a_{1}} p_{2}^{-a_{2}} \cdots p_{s}^{-a_{s}} \alpha^{n-m}-1\right|<\frac{4}{\alpha^{m}} .
$$


A continuación, aplicamos el Teorema 2.1 con $t:=s+1$ logaritmos. Para esto, tomamos

$$
\eta_{i}:=p_{i}, \quad b_{i}:=-a_{i}, \quad \text { para } \quad 1 \leq i \leq s \quad \text { y } \quad \eta_{s+1}:=\alpha, \quad b_{s+1}:=n-m .
$$

Así, $\Lambda_{1}:=p_{1}^{-a_{1}} p_{2}^{-a_{2}} \cdots p_{s}^{-a_{s}} \alpha^{n-m}-1$, y por $(6)$,

$$
\left|\Lambda_{1}\right|<\frac{4}{\alpha^{m}}
$$

El cuerpo de números algebraicos $\mathbb{K}:=\mathbb{Q}(\alpha)$ contiene los $\eta_{i}$ y tiene grado 3 sobre $\mathbb{Q}$; así, $D:=3$. Además, podemos tomar $B:=n$. Ahora, fácilmente podemos ver que $h\left(p_{i}\right)=\log p_{i}$, para $i=1,2, \ldots, s$, y dado que $\alpha$ es un entero algebraico con raíces conjugadas subunitarias (es decir, con norma compleja menor que 1), podemos concluir que $h(\alpha)<(\log \alpha) / 3$. Así, tomamos

$$
A_{i}:=3 \log p_{i}, \quad \text { para } 1 \leq i \leq s \quad \text { y } \quad A_{s+1}:=\log \alpha .
$$

Con el fin de usar la conclusión del teorema de Matveev, nos resta ver que $\Lambda_{1}$ no es cero. En efecto, en caso contrario obtendríamos que

$$
\alpha^{n-m}=p_{1}^{a_{1}} p_{2}^{a_{2}} \cdots p_{s}^{a_{s}} .
$$

Conjugando la igualdad anterior por un automorfismo $\sigma \in G$ tal que $\sigma(\alpha)=\beta$ y tomando valor absoluto, tenemos

$$
|\beta|^{n-m}=p_{1}^{a_{1}} p_{2}^{a_{2}} \cdots p_{s}^{a_{s}}
$$

sin embargo esto no es posible, dado que $|\beta|<1$ y $n-m>0$, mientras que $p_{1}^{a_{1}} p_{2}^{a_{2}} \cdots p_{s}^{a_{s}}>1$. Así, $\Lambda_{1} \neq 0$.

La conclusión del Teorema 2.1 nos da la siguiente cota inferior para $\log \left|\Lambda_{1}\right|$ :

$$
-1,4 \times 30^{s+4}(s+1)^{4,5} 3^{s+2}(1+\log 3)(1+\log n)(\log \alpha) \cdot \prod_{i=1}^{s} \log p_{i} .
$$

Combinando con la desigualdad $\log \left|\Lambda_{1}\right|<\log 4-m \log \alpha$, la cual obtenemos desde (7), concluimos que

$$
m<2,5 \times 10^{7} g_{1}(s) \log n, \quad \text { con } g_{1}(s)=90^{s}(s+1)^{4,5} \cdot \prod_{i=1}^{s} \log p_{i} .
$$

Retornando a la ecuación (4), sustituimos únicamente $T_{n}$ de acuerdo con la identidad (2) y realizamos las operaciones algebraicas correspondientes, para obtener

$$
\left|\Lambda_{2}\right|:=\left|p_{1}^{a_{1}} p_{2}^{a_{2}} \cdots p_{s}^{a_{s}} T_{m} d_{\alpha}^{-1} \alpha^{-(n-1)}-1\right|<\frac{2}{\alpha^{n}} .
$$

Aplicamos una vez más el método de las formas lineales con $t:=s+3$ logaritmos y con los parámetros

$$
\eta_{i}:=p_{i}, \quad b_{i}:=a_{i} \quad \text { para } \quad 1 \leq i \leq s, \quad \eta_{s+1}:=T_{m}, \quad b_{s+1}:=1,
$$

Vol. 33, No. 2, 2015] 


$$
\eta_{s+2}:=d_{\alpha}, \quad b_{s+2}:=-1, \quad \eta_{s+3}:=\alpha, \quad b_{s+3}:=-(n-1) .
$$

Como en la primera aplicación del Teorema 2.1 , tomamos $\mathbb{K}:=\mathbb{Q}(\alpha), D:=3, B:=n$, $A_{i}:=3 \log p_{i}$, para $i=1, \ldots, s$, y $A_{s+3}:=\log \alpha$. Ahora, por la desigualdad (3), concluimos que $h\left(T_{m}\right)=\log T_{m}<m \log \alpha$, así que tomamos $A_{s+1}:=3 m \log \alpha$. En cuanto a $\eta_{s+2}$, notamos que su polinomio primitivo minimal sobre los enteros está dado por

$$
44 \cdot \prod_{\sigma \in G}\left(X-\sigma\left(d_{\alpha}\right)\right)=44 X^{3}-44 X^{2}+12 X-1 .
$$

Así, en vista de que $\left|d_{\alpha}\right|,\left|d_{\beta}\right|,\left|d_{\gamma}\right|<1$, tenemos que $h\left(d_{\alpha}\right)=(\log 44) / 3$. Por lo tanto, tomamos $A_{s+2}:=\log 44$. Veamos que $\Lambda_{2} \neq 0$. En el caso de ser cero, $d_{\alpha} \alpha^{n-1}=$ $p_{1}^{a_{1}} p_{2}^{a_{2}} \cdots p_{s}^{a_{s}} T_{m}$ es un entero no nulo. Sin embargo, conjugando esta igualdad por un automorfismo $\sigma \in G$ tal que $\sigma(\alpha)=\beta$, obtenemos que

$$
p_{1}^{a_{1}} p_{2}^{a_{2}} \cdots p_{s}^{a_{s}} T_{m}=\sigma\left(p_{1}^{a_{1}} p_{2}^{a_{2}} \cdots p_{s}^{a_{s}} T_{m}\right)=\sigma\left(d_{\alpha} \alpha^{n-1}\right)=d_{\beta} \beta^{n-1} .
$$

Tomando valor absoluto en ambos lados de la igualdad anterior, obtenemos una contradicción (basta notar que $d_{\beta}$ y $\beta$ son subunitarios).

A partir de la conclusión del Teorema 2.1 y de la desigualdad (9), obtenemos que

$$
\exp \left(-6 \times 10^{10} g_{2}(s)(\log n) m\right)<\left|\Lambda_{2}\right|<\frac{2}{\alpha^{n}},
$$

donde $g_{2}(s)=90^{s}(s+3)^{4,5} \cdot \prod_{i=1}^{s} \log p_{i}$. Tomando logaritmo en ambos lados de la desigualdad (10), e incluyendo la cota superior para $m$ dada por (8), concluimos que

$$
\frac{n}{\log ^{2} n}<2,4 \times 10^{18} g_{3}(s), \quad \text { con } \quad g_{3}(s)=90^{2 s}\left(s^{2}+4 s+3\right)^{4,5} \cdot \prod_{i=1}^{s} \log ^{2} p_{i} .
$$

Con el fin de obtener una cota para $n$, presentamos el siguiente argumento analítico, el cual aplicaremos en la desigualdad (11).

Lema 3.1. Sean $h \geq 1$ un entero positivo y $A \geq 2(h+1) \log (h+1)$.

$$
\text { Si } \frac{x}{(\log x)^{h}}<A, \quad \text { entonces } \quad x<(h+1)^{h} A(\log A)^{h} .
$$

Demostración. Para el caso $h=1$ (ver [22]). Asumiremos que $h \geq 2$. Notemos que la función $x \mapsto x /(\log x)^{h}$ es creciente para $x>e^{h}$. Argumentando por contradicción, supongamos que $x \geq(h+1)^{h} A(\log A)^{h}$; entonces $x>e^{h}$, dado que $A>e$. Por lo tanto,

$$
A>\frac{x}{(\log x)^{h}} \geq \frac{(h+1)^{h} A(\log A)^{h}}{\left(\log \left((h+1)^{h} A(\log A)^{h}\right)\right)^{h}} .
$$

Realizando las simplificaciones respectivas, obtenemos que $A / \log A<h+1$, y aplicando el caso $h=1$ concluimos que $A<2(h+1) \log (h+1)$, lo cual no es cierto.

Aplicando el Lema 3.1 en la desigualdad (11), con $A:=2,4 \times 10^{18} g_{3}(s)$ concluimos que

$$
n<2,2 \times 10^{20} g_{5}(s), \quad \text { con } g_{5}=g_{3}(s) g_{4}(s),
$$

donde $g_{4}(s)=\log ^{2}\left(2,4 \times 10^{18} g_{3}(s)\right)$. Con esto concluimos la prueba del Teorema 1.1. 


\section{Resultado numérico}

En esta sección determinamos todos los cocientes de números Tribonacci que son $\{2,3,7,103\}$-unidades; en otras palabras, estamos interesados en hallar todas las soluciones de la ecuación diofántica

$$
T_{n}=2^{a} 3^{b} 7^{c} 103^{d} T_{m}, \quad \text { con } \quad n>m \geq 2 \quad \text { y } \quad a, b, c, d \in \mathbb{Z} .
$$

Notemos que para $s=4$ y $S=\{2,3,7,103\}$, los cálculos realizados en la sección anterior nos permiten concluir que

$$
\begin{gathered}
g_{1}(4)=6,3 \times 10^{11}, \quad g_{2}(4)=3 \times 10^{12}, \quad g_{3}(4)=2 \times 10^{24}, \\
g_{4}(4)=10^{4}, \quad g_{5}(4)=1,8 \times 10^{28} .
\end{gathered}
$$

Así, deducimos de (8) y (12) que

$$
m<1,6 \times 10^{19} \quad \text { y } \quad n<4 \times 10^{48} .
$$

A continuación reducimos las cotas para $m$ y $n$ dadas anteriormente, mediante el uso de la teoría de retículos.

Retornando a la desigualdad (7) (para $s$ y $S$ dados en esta sección), tomamos

$$
\Gamma_{1}:=-a \log 2-b \log 3-c \log 7-d \log 103+(n-m) \log \alpha .
$$

Así, $\Lambda_{1}=e^{\Gamma_{1}}-1$. Supongamos que $m \geq 5$; entonces por (7) tenemos que

$$
\left|\Lambda_{1}\right|=\left|e^{\Gamma_{1}}-1\right|<\frac{4}{\alpha^{m}}<\frac{1}{5} .
$$

De este modo, $e^{\left|\Gamma_{1}\right|}<5 / 4$, y por (15) concluimos que

$$
\left|\Gamma_{1}\right| \leq e^{\left|\Gamma_{1}\right|}\left|e^{\Gamma_{1}}-1\right|<\frac{5}{\alpha^{m}} .
$$

Por lo tanto,

$$
|a \log 2+b \log 3+c \log 7+d \log 103-(n-m) \log \alpha|<5 \times \alpha^{-m} .
$$

Ahora procedemos a estimar una cota inferior para $\left|\Gamma_{1}\right|$. En este punto de nuestro trabajo usamos un resultado de la geometría de números sobre una cota inferior para formas lineales con coeficientes enteros acotados. Sean $\alpha_{1}, \ldots, \alpha_{t} \in \mathbb{R}$. Consideremos el retículo entero $\Omega=\left\langle\mathbf{b}_{1}, \ldots, \mathbf{b}_{t}\right\rangle_{\mathbb{Z}}$, con vectores $\mathbf{b}_{j}$ dados por

$$
\mathbf{b}_{j}=\mathbf{e}_{j}+\left\lfloor C \alpha_{j}\right\rceil \mathbf{e}_{t} \text { para } 1 \leq j \leq t-1 \quad \text { y } \quad \mathbf{b}_{t}=\left\lfloor C \alpha_{t}\right\rceil \mathbf{e}_{t},
$$

donde $C$ es una constante positiva suficientemente grande.

Vol. 33, No. 2, 2015] 
Lema 4.1. Sean, $X_{1}, \ldots, X_{t}$ enteros positivos tales que $X:=\operatorname{máx}\left\{X_{i}\right\}$ y $C>(t X)^{t}$ una constante fija. Con la notación anterior sobre $\Omega$, consideremos una base reducida $\left\{\mathbf{b}_{i}\right\}$ para $\Omega$ y su base de Gram-Schmidt $\left\{\mathbf{b}_{i}^{*}\right\}$ asociada. Fijamos las constantes

$$
c_{1}=\operatorname{máx}_{1 \leq i \leq t} \frac{\left\|\mathbf{b}_{1}\right\|}{\left\|\mathbf{b}_{i}^{*}\right\|}, \quad \delta=\frac{\left\|\mathbf{b}_{1}\right\|}{c_{1}}, \quad Q=\sum_{i=1}^{t-1} X_{i}^{2} \quad y \quad T=\sum_{i=1}^{t} X_{i} / 2 .
$$

Si los enteros $x_{i}$ cumplen que $\left|x_{i}\right| \leq X_{i}$, para $i=1, \ldots, t$ y $\delta^{2} \geq T^{2}+Q$, entonces tenemos que

$$
\left|\sum_{i=1}^{t} x_{i} \alpha_{i}\right| \geq \frac{\sqrt{\delta^{2}-Q}-T}{C}
$$

Para más detalles, consultar [6, Sección 2.3.5].

Antes de aplicar el Lema 4.1 en $\Gamma_{1}$, notemos que $\Gamma_{1} \neq 0$, dado que $\Lambda_{1} \neq 0$. Fijamos $C:=(6 X)^{5}$, donde $X:=4 \times 10^{48}$ es una cota superior para máx $\{|a|,|b|,|c|,|d|, n-m\}$ (de acuerdo con 14), y consideramos el retículo $\Omega_{1}$ generado por

$$
\begin{aligned}
& b_{1}:=(1,0,0,0,\lfloor C \log 2\rfloor), \quad b_{2}:=(0,1,0,0,\lfloor C \log 3\rfloor), \\
& b_{3}:=(0,0,1,0,\lfloor C \log 7\rfloor), \\
& b_{4}:=(0,0,0,1,\lfloor C \log 103\rfloor), \quad b_{5}:=(0,0,0,1,\lfloor C \log \alpha\rfloor) .
\end{aligned}
$$

Usando Mathematica ${ }^{\circledR}$ estimamos una base reducida $\left\{\mathbf{b}_{i}\right\}$ (algoritmo LLL) para $\Omega_{1}$ y su base de Gram-Schmidt $\left\{\mathbf{b}_{i}^{*}\right\}$ asociada. Con la anterior información, encontramos que

$$
\begin{array}{cc}
c_{1}=1,212898017 \ldots, & \delta=1,6 \times 10^{53}, \\
Q \leq 5 X^{2}=8 \times 10^{97} \quad \text { y } & T \leq 3 X=1,2 \times 10^{49} .
\end{array}
$$

Dado que $\delta^{2}-\left(T^{2}+Q\right)>10^{97}$, desde la conclusión del Lema 4.1 obtenemos que

$$
\left|\Gamma_{1}\right|>\frac{\sqrt{\delta^{2}-Q}-T}{C}>5,6 \times 10^{-200},
$$

y combinando esta desigualdad con (16) concluimos que $m \leq 760$.

Volviendo a la desigualdad (10) con nuestra nueva cota en $m$, obtenemos la desigualdad

$$
n<2,3 \times 10^{26} \log n,
$$

de la cual se sigue que $n<2 \times 10^{28}$. Ahora, con esta nueva cota para $n$, aplicamos de nuevo el Lema 4.1 para obtener una nueva cota inferior para $\left|\Gamma_{1}\right|$, la cual combinamos con (16):

$$
2,9 \times 10^{-118}<\left|\Gamma_{1}\right|<5 \times \alpha^{-m} .
$$

Esta vez tenemos que $m \leq 450$, y en consecuencia $n<8,6 \times 10^{27}$.

Ahora vamos a la desigualdad (9) (para $s$ y $S$ dados en esta sección) y tomamos

$$
\Gamma_{2}:=a \log 2+b \log 3+c \log 7+d \log 103+\log \left(T_{m} d_{\alpha}^{-1}\right)-(n-1) \log \alpha .
$$


Entonces, $\Lambda_{2}=e^{\Gamma_{2}}-1$. Por (9) y el hecho de que $n \geq 3$, concluimos que $\left|\Lambda_{2}\right|<1 / 3$. Luego $e^{\left|\Gamma_{2}\right|}<3 / 2$, y así $\left|\Gamma_{2}\right|<3 / \alpha^{n}$. Por lo tanto,

$$
\left|a \log 2+b \log 3+c \log 7+d \log 103+\log \left(T_{m} d_{\alpha}^{-1}\right)-(n-1) \log \alpha\right|<3 \times \alpha^{-n} .
$$

Notemos que $\Gamma_{2} \neq 0$, porque $\Lambda_{2} \neq 0$. Para cada $m \in[2,450]$ tomamos $X:=8,6 \times 10^{27}$ (última cota para $n$ ), $C:=(9 X)^{9}$ y fijamos la base entera para el retículo $\Omega_{2}$ respectivo. Mediante el uso de Mathematica ${ }^{\circledR}$ implementamos los cálculos descritos en el Lema 4.1, para obtener la siguiente cota inferior $\left|\Gamma_{2}\right|>10^{-218}$, la cual, combinada con (18), conduce a $n \leq 825$. Con esta nueva cota para $n$ en lugar de la cota dada en (14), retornamos a la desigualdad (16) e implementamos una vez más el procedimiento de reducción mediante el algoritmo LLL (Lema 4.1). Esta vez, tomando $X:=825$ y $C:=(6 X)^{6}$, obtenemos que $\left|\Gamma_{1}\right|>6,4 \times 10^{-19}$, y así, $m \leq 75$. Con esta pequeña cota para $m$, vamos a la desigualdad (18), donde para cada $m \in[2,75]$ procedemos como antes, con $X:=825 \mathrm{y}$ $C:=(10 X)^{9}$. Pocos segundos después, Mathematica ${ }^{\circledR}$ revela que $\left|\Gamma_{2}\right|>9,7 \times 10^{-31}, \mathrm{y}$ como consecuencia de (18) tenemos que $n \leq 120$.

Realizando una última vez el ciclo de reducción que involucra las desigualdades (16), (18) y el Lema 4.1, obtenemos que

$$
m \in[2,60] \quad \text { y } \quad n \in[m+1,100] .
$$

Listando la factorización de $T_{n}$, con $n \in[3,100]$, podemos notar que para $n \geq 19$, cada número $T_{n}$ tiene un divisor primitivo mayor que 7 y distinto de 103; de hecho, para $n \geq 37, T_{n}$ tiene un divisor primitivo mayor que 103 (en general en una sucesión de enteros $\left\{u_{n}\right\}_{n \geq 0}$, decimos que un término $u_{n}$ tiene un divisor primitivo si $u_{n}$ tiene un factor primo que no divide a $u_{m}$, para todo $m<n$ ). Por lo tanto, para $n \geq 19$ la ecuación (13) no tiene soluciones. De esta manera, nuestra búsqueda se reduce a $m<n \leq 18$.

Las soluciones halladas las clasificaremos de acuerdo con $m$ :

- $m=2,3, T_{2}=T_{3}=1 \mathrm{y}$

$$
\begin{array}{lll}
T_{4}=2, & T_{5}=2^{2}, \quad T_{6}=7, & T_{8}=2^{3} \cdot 3, \\
T_{10}=3^{4}, & T_{13}=2^{3} \cdot 3^{2} \cdot 7, & T_{14}=3^{2} \cdot 103, \\
T_{16}=2^{6} \cdot 7^{2}, & T_{17}=2^{3} \cdot 7 \cdot 103, & T_{18}=103^{2} .
\end{array}
$$

- $m=4, T_{4}=2 \mathrm{y}$

$$
\begin{array}{lll}
T_{5}=2 T_{4}, & T_{6}=2^{-1} \cdot 7 T_{4}, & T_{8}=2^{2} \cdot 3 T_{4}, \\
T_{10}=2^{-1} \cdot 3^{4} T_{4}, & T_{13}=2^{2} \cdot 3^{2} \cdot 7 T_{4}, & T_{14}=2^{-1} \cdot 3^{2} \cdot 103 T_{4}, \\
T_{16}=2^{5} \cdot 7^{2} T_{4}, & T_{17}=2^{2} \cdot 7 \cdot 103 T_{4}, & T_{18}=2^{-1} \cdot 103^{2} T_{4} .
\end{array}
$$

- $m=5, T_{5}=4 \mathrm{y}$

$$
\begin{array}{ccc}
T_{6}=2^{-2} \cdot 7 T_{5}, & T_{8}=2 \cdot 3 T_{5}, & T_{10}=2^{-2} \cdot 3^{4} T_{5}, \\
T_{13}=2 \cdot 3^{2} \cdot 7 T_{5}, & T_{14}=2^{-2} \cdot 3^{2} \cdot 103 T_{5}, & T_{16}=2^{4} \cdot 7^{2} T_{5}, \\
T_{17}=2 \cdot 7 \cdot 103 T_{5}, & T_{18}=2^{-2} \cdot 103^{2} T_{5} .
\end{array}
$$

Vol. 33, No. 2, 2015] 
- $m=6, T_{6}=7 \mathrm{y}$

$$
\begin{array}{lll}
T_{8}=2^{3} \cdot 3 \cdot 7^{-1} T_{6}, & & T_{10}=3^{4} \cdot 7^{-1} T_{6}, \\
T_{13}=2^{3} \cdot 3^{2} T_{6}, & T_{14}=3^{2} \cdot 7^{-1} \cdot 103 T_{6}, & T_{16}=2^{6} \cdot 7 T_{6}, \\
T_{17}=2^{3} \cdot 103 T_{6}, & & T_{13}=7^{-1} \cdot 103^{2} T_{6} .
\end{array}
$$

- $m=8, T_{8}=2^{3} \cdot 3 \mathrm{y}$

$$
\begin{array}{lll}
T_{10}=2^{-3} \cdot 3^{2} T_{6}, & T_{13}=3 \cdot 7 T_{8}, & T_{14}=2^{-3} \cdot 3 \cdot 103 T_{8}, \\
T_{16}=2^{3} \cdot 3^{-1} \cdot 7^{2} T_{8}, & T_{17}=3^{-1} \cdot 7 \cdot 103 T_{8}, & T_{18}=2^{-3} \cdot 3^{-1} \cdot 103^{2} T_{8} .
\end{array}
$$

- $m=10, T_{10}=3^{4} \mathrm{y}$

$$
\begin{gathered}
T_{13}=2^{3} \cdot 3^{-2} \cdot 7 T_{10}, \quad T_{14}=3^{-2} \cdot 103 T_{10}, \quad T_{16}=2^{6} \cdot 3^{-4} \cdot 7^{2} T_{10}, \\
T_{17}=2^{3} \cdot 3^{-4} \cdot 7 \cdot 103 T_{10}, \quad T_{18}=3^{-4} \cdot 103^{2} T_{10} .
\end{gathered}
$$

- $m=13, T_{13}=2^{3} \cdot 3^{2} \cdot 7 \mathrm{y}$

$$
\begin{array}{ll}
T_{14}=2^{-3} \cdot 7^{-1} \cdot 103 T_{13}, & T_{16}=2^{3} \cdot 3^{-2} \cdot 7 T_{13}, \\
T_{17}=3^{-2} \cdot 103 T_{13}, & T_{18}=2^{-3} \cdot 3^{-2} \cdot 7^{-1} \cdot 103 T_{13} .
\end{array}
$$

- $m=14, T_{14}=3^{2} \cdot 103 \mathrm{y}$

$$
T_{16}=2^{6} \cdot 3^{-2} \cdot 7^{2} \cdot 103^{-1} T_{14}, \quad T_{17}=2^{3} \cdot 3^{-2} \cdot 7 T_{14}, \quad T_{18}=3^{-2} \cdot 103 T_{14} .
$$

- $m=16, T_{16}=2^{6} \cdot 7^{2}$ y $T_{17}=2^{-3} \cdot 7^{-1} \cdot 103 T_{16}, \quad T_{18}=2^{-6} \cdot 7^{-2} \cdot 103^{2} T_{16}$.

- $m=17, T_{17}=2^{3} \cdot 7 \cdot 103$ y $T_{18}=2^{-3} \cdot 7^{-1} \cdot 103 T_{17}$.

\section{Triplas diofánticas}

Una $m$-tupla diofántica es un conjunto $\left\{a_{1}, \ldots, a_{m}\right\}$ de números racionales o enteros positivos, con la propiedad de que el producto de cualesquiera dos de sus elementos (distintos) es un cuadrado menos uno, es decir, tal que $a_{i} a_{j}+1$ es un cuadrado para todo $1 \leq i<j \leq m$. Es bien conocido que Diofanto presentó la cuádrupla racional $\{1 / 16,33 / 16,17 / 4,105 / 16\}$, y que mucho tiempo después Fermat encontró la cuádrupla entera $\{1,3,8,120\}$.

En el caso entero, Euler dio una familia infinita de cuádruplas diofánticas:

$$
\{a, b, a+b+2 t, 4 t(t+a)(t+b)\}, \text { donde } a b+1=t^{2} .
$$

Por otro lado, Arkin, Hoggatt y Strauss [1] dieron una manera de extender cualquier tripla diofántica a una cuádrupla diofántica. Más precisamente, si $\{a, b, c\}$ es una tripla diofántica con $a b+1=t^{2}, \quad a c+1=u^{2}, \quad b c+1=v^{2}$, con $t, u, v$ enteros positivos, entonces $\{a, b, c, d\}$ es una cuádrupla diofántica, donde $d=a+b+c+2 a b c+2 t u v$. Respecto a $m$-tuplas diofánticas, con $m \geq 5$, Baker y Davenport mostraron en [2] que 
la cuádrupla diofántica $\{1,3,8,120\}$ no puede ser extendida a una quíntupla diofántica. Recientemente Dujella [8] mostró que no existen séxtuplas diofánticas, y que hay a lo más una cantidad finita de quíntuplas diofánticas. Poco después, él mismo presentó en [9] la cota superior explícita $10^{1930}$ para el número de quíntuplas diofánticas. Esta cota fue reducida sustancialmente a $6,8 \times 10^{32}$ por Elsholtz, Filipin y Fujita [10]. Sin embargo, en el caso racional no es conocido ni siquiera si $m$ debe ser acotada. Gibbs [13] encontró algunos ejemplos con $m=6$.

Una generalización natural de este problema consiste en reemplazar los cuadrados por miembros de una sucesión entera. Sea $\mathbf{U}:=\left\{U_{n}\right\}_{n \geq 0}$ una sucesión de enteros; diremos que un conjunto $\left\{a_{1}, \ldots, a_{m}\right\}$ de enteros positivos es una $m$-tupla diofántica asociada a U si $a_{i} a_{j}+1$ es un miembro de $U$, para todo $1 \leq i<j \leq m$. Asumiremos que $m \geq 3$ para evitar trivialidades. En [3],[4],[17],[18],[19] se estudiaron $m$-tuplas diofánticas asociadas a potencias superiores con exponente fijo o variable, mientras que Fuchs, Luca y Szalay investigaron en [11] sobre $m$-tuplas diofánticas con miembros en sucesiones lineales binarias no degeneradas. Particularmente, Luca y Szalay mostraron que no existen triplas diofánticas con valores en la sucesión de Fibonacci (ver [20]), y que la única tripla diofántica con valores en la sucesión de Lucas es $\{a, b, c\}=\{1,2,3\}$ (ver [21]). Muy poco se conoce acerca de $m$-tuplas diofánticas asociadas a sucesiones lineales recurrentes de order mayor que dos. En trabajo conjunto con F. Luca, hemos tratado con la sucesión Tribonacci $T$, mostrando en [15] que no existen cuádruplas diofánticas asociadas a $T$. Recientemente, Fuchs, Luca y Szalay mostraron que existe a lo más una cantidad finita de triplas diofánticas $1<a<b<c$ asociadas a cada sucesión $k$-generalizada de Fibonacci, donde para $k=3$ tenemos la sucesión Tribonacci (ver [12]). Sin embargo, el problema de determinar todas las triplas diofánticas asociadas a $T$ continúa abierto (hasta el momento no conocemos un ejemplo). También, en trabajo conjunto con F. Luca, hemos probado que no existen cuádruplas diofánticas asociadas a la sucesión $T+1$, es decir que no existen $m$-tuplas $\left\{a_{1}, \ldots, a_{m}\right\}$ de enteros positivos tales que $a_{i} a_{j} \in T$, con $m \geq 4$ (consultar [16]). Estudiando la ecuación diofántica

$$
a T_{n}=b T_{m},
$$

en enteros positivos $a, b, n, m$ con $a<b$ primos relativos en un rango pequeño, obtuvimos mediante búsqueda computacional que para $a, b \in\{9,56,103\}$ la ecuación (19) siempre tiene solución. Curiosamente $\{9,56,103\}$ es una tripla diofántica asociada a $T+1$, dado que

$$
9 \times 56=T_{13}, \quad 9 \times 103=T_{14}, \quad 56 \times 103=T_{17} .
$$

En esta sección nos proponemos mostrar que cualquier par de miembros de la tripla diofántica $\{9,56,103\}$, asociada a $T+1$, no puede ser extendida a otra tripla diofántica asociada a $T+1$. Primero que todo, notemos que si $a, b \in\{9,56,103\}$ y $\{a, b, x\}$ es una tripla diofántica asociada a $T+1$, con $a<b<x$, entonces en particular tenemos que

$$
a x=T_{n}, \quad b x=T_{m}, \quad \text { para algún } n, m \geq 1 .
$$

Eliminando $x$ en (20), obtenemos

$$
T_{n}=(b / a) T_{m}, \quad \text { donde } b / a \text { es una }\{2,3,7,103\} \text {-unidad. }
$$

Las soluciones que encontramos en la sección anterior para la ecuación diofántica (13) nos permiten concluir que la única solución, cuando $a=9, b=56$, es $x=103$; y que

Vol. 33, No. 2, 2015] 
en los demás casos no hay solución para (20). Así, ninguna dupla $\{a, b\} \subseteq\{9,56,103\}$ la podemos extender a una tripla diofántica asociada a $T+1$ distinta a $\{9,56,103\}$.

Finalizamos con la siguiente proposición, la cual es evidente.

Proposición 5.1. Sean a y $b$ enteros positivos tales que $a b \in T$. La ecuación diofántica $a T_{n}=b T_{m}$ tiene solución si y solo si existe un entero positivo $x$ tal que el conjunto $\{a, b, x\}$ es una tripla diofántica asociada a $T+1$.

\section{Agradecimientos}

El autor agradece a los evaluadores de este artículo por los comentarios, los cuales mejoraron la calidad de este documento. El autor agradece a la Universidad del Valle por su apoyo durante sus estudios de Doctorado.

\section{Referencias}

[1] Arkin J., Hoggatt V.E. and Strauss E.G., "On Euler's solution of a problem of Diophantus", Fibonacci Quart. 17 (1979), No. 4, 333-339.

[2] Baker A. and Davenport H., "The equations $3 x^{2}-2=y^{2}$ and $8 x^{2}-7=z^{2 ",}$ Quart. J. Math. Oxford Ser. (2) 20 (1969), 129-137.

[3] Bugeaud Y. and Dujella A., "On a problem of Diophantus for higher powers", Math. Proc. Cambridge Philos. Soc. 135 (2003), No. 1, 1-10.

[4] Bugeaud Y. and Gyarmati K., "On generalizations of a problem of Diophantus", Illinois J. Math. 48 (2004), No. 4, 1105-1115.

[5] Bravo J.J. and Luca F., "Powers of two in generalized Fibonacci sequences", Rev. Colombiana Mat. 46 (2012), No. 1, 67-79.

[6] Cohen H., Number Theory, Vol. I: Tools and Diophantine equations, Springer, New York, 2007.

[7] Dresden G.P.B. and Du Z., "A simplified Binet formula for $k$-generalized Fibonacci numbers", J. Integer Seq. 17 (2014), No. 4, Article 14.4.7, 9 pp.

[8] Dujella A., "There are only finitely many Diophantine quintuples", J. Reine Angew. Math. 566 (2004), 183-214.

[9] Dujella A., "On the number of Diophantine m-tuples", Ramanujan J. 15 (2008), No. 1, $37-46$.

[10] Elsholtz C., Filipin A. and Fujita Y., "On Diophantine quintuples and $D(-1)$-quadruples", Monatsh. Math. 175 (2014), No. 2, 227-239.

[11] Fuchs C., Luca F. and Szalay L., "Diophantine triples with values in binary recurrences", Ann. Sc. Norm. Super. Pisa Cl. Sc. (5) 7 (2008), No. 4, 579-608.

[12] Fuchs C., Luca F. and Szalay L., "Diophantine triples with values in $k$-generalized Fibonacci sequences" (preprint).

[13] Gibbs P., "Some rational Diophantine sextuples", Glas. Mat. Ser. III 41 (2006), No. 2, 195-203.

[Revista Integración 
[14] Gómez Ruiz C.A. and Luca F., "On the largest prime factor of the ratio of two generalized Fibonacci numbers", J. Number Theory 152 (2015), 182-203.

[15] Gómez Ruiz C.A. and Luca F., "Tribonacci Diophantine quadruples", Glas. Mat. Ser. III 50 (2015), No. 1, 17-24.

[16] Gómez Ruiz C.A. and Luca F., "Diophantine quadruples in the sequence of shifted Tribonacci numbers", Publ. Math. Debrecen 86 (2015) No. 3-4, 473-491.

[17] Gyarmati K., Sárközy A. and Stewart C.L., "On shifted products which are powers", $M a-$ thematika 49 (2002), No. 1-2, 227-230.

[18] Gyarmati K. and Stewart C.L., "On powers in shifted products", Glas. Mat. Ser. III 42 (2007), No. 2, 273-279.

[19] Luca F., "On shifted products which are powers", Glas. Mat. Ser. III 40 (2005), No. 1, 13-20.

[20] Luca F. and Szalay L., "Fibonacci Diophantine triples", Glas. Mat. Ser. III 43 (2008), No. $2,253-264$.

[21] Luca F. and Szalay L., "Lucas Diophantine triples", Integers 9 (2009), 441-457.

[22] Luca F. and Oyono R., "An exponential Diophantine equation related to powers of two consecutive Fibonacci numbers", Proc. Japan Acad. Ser. A Math. Sci. 87 (2011), No. 4, 45-50.

[23] Lucas E., "Théorie des fonctions numériques simplement périodiques", Amer. J. Math. 1 (1878), No. 2-3, 184-240.

[24] Matveev E.M., "An explicit lower bound for a homogeneous rational linear form in the logarithms of algebraic numbers II", Izv. Math. 64 (2000), No. 6, 1217-1269.

[25] Spickerman W.R., "Binet's formula for the Tribonacci sequence", Fibonacci Quart. 20 (1982), No. 2, 118-120. 